\title{
ERRATA
}

\section{Erratum: Parametric excitation of spin waves in uniaxial ferrites [Tech. Phys. 43, 539 (1998)]}

A. V. Nazarov and A. G. Gurevich

Zh. Tekh. Fiz. 69, 143 (August 1999)

[S1063-7842(99)03008-1]

Formula (9) should read:

$M_{z}=M_{0}-\frac{m_{+} m_{-}}{2 M_{0}}$.

Formula (12) should read:

$\omega_{H}=\gamma H_{c 0} \cos \left(\theta_{H}-\theta_{0}\right)$.

Formula (24) should read:

$$
\begin{aligned}
Y_{k}= & \lambda_{k}^{2}\left(-\omega_{M} \frac{k_{+} k_{z}}{k^{2}}-i \frac{\omega_{M}}{4 \pi} N_{23}^{a}\right) a_{0}^{+}+\mu_{k}^{2}\left(-\omega_{M} \frac{k_{+}^{*} k_{z}}{k^{2}}+i \frac{\omega_{M}}{4 \pi} N_{23}^{a}\right) a_{0}^{-*}-\lambda_{k} \mu_{k} \\
& \times\left(2 \gamma h_{z}-a_{0}^{+} \omega_{M} \frac{k_{+} k_{z}}{k^{2}}-a_{0}^{-*} \omega_{M} \frac{k_{+} k_{z}}{k^{2}}+i \frac{\omega_{M}}{2 \pi} N_{23}^{a}\left(a_{0}^{+}-a_{0}^{-*}\right)\right) .
\end{aligned}
$$

Formula (27) should read:

$$
\begin{aligned}
W_{k}= & \frac{1}{4}\left(A_{k}+\xi_{a}+\omega_{k}\right)\left(2 \exp \left(i \varphi_{k}\right) \sin 2 \theta_{k}+i \frac{N_{23}^{a}}{\pi}\right) a_{01}+\frac{1}{4}\left(A_{k}+\xi_{a}-\omega_{k}\right) \\
& \times \frac{\left[B_{k} \exp \left(2 i \varphi_{k}\right)-\eta_{a}\right]^{2}}{\left|B_{k} \exp \left(2 i \varphi_{k}\right)-\eta_{a}\right|^{2}}\left(2 \exp \left(-i \varphi_{k}\right) \sin 2 \theta_{k}-i \frac{N_{23}^{a}}{\pi}\right) a_{02}+\left[B_{k} \exp \left(2 i \varphi_{k}\right)-\eta_{a}\right] \\
& \times\left[\frac{\cos \theta_{h}}{\omega_{M}}-\frac{a_{01}}{2} \exp \left(-i \varphi_{k}\right) \sin 2 \theta_{k}-\frac{a_{02}}{2} \exp \left(i \varphi_{k}\right) \sin 2 \theta_{k}+i \frac{N_{23}^{a}}{2 \pi}\left(a_{0}^{+}-a_{0}^{-*}\right)\right] .
\end{aligned}
$$

Formula (30) should read:

$h_{\mathrm{thr}}=\min \left\{\frac{\Delta H_{k}}{4 \pi M_{0} \sin ^{2} \theta_{k} \exp \left(2 i \varphi_{k}\right)+2 H_{\mathrm{A} 1} \sin ^{2} \theta_{0}}\right\} \frac{\omega_{k}}{\cos \theta_{h}}$.

Translated by R. M. Durham 